\title{
Detecting genuine multipartite entanglement of pure states with bipartite correlations
}

\author{
Marcin Markiewicz, ${ }^{1}$ Wiesław Laskowski, ${ }^{1}$ Tomasz Paterek, ${ }^{2,3}$ and Marek Żukowski ${ }^{1}$ \\ ${ }^{1}$ Institute of Theoretical Physics and Astrophysics, University of Gdańsk, 80-952 Gdańsk, Poland \\ ${ }^{2}$ School of Physical and Mathematical Sciences, Nanyang Technological University, Singapore \\ ${ }^{3}$ Centre for Quantum Technologies, National University of Singapore, Singapore
}

(Received 18 September 2012; published 26 March 2013)

\begin{abstract}
Monogamy of bipartite correlations leads, for arbitrary pure multiqubit states, to simple conditions able to indicate various types of multipartite entanglement by being capable of excluding the possibility of $k$ separability.
\end{abstract}

DOI: 10.1103/PhysRevA.87.034301

PACS number(s): 03.67.Mn

For bipartite systems the phenomenon of quantum entanglement [1,2] manifests itself in correlations. One might expect that genuinely $n$-partite entanglement gives rise to nonvanishing correlations between all $n$ subsystems. This is incorrect, at least when correlations are quantified as average values of a product of local measurement results $[3,4]$ (for a discussion on quantum correlations without classical correlations, see, e.g., Ref. [5]). Thus, in order to detect genuine multipartite entanglement of certain states one has to rely on correlations between smaller numbers of subsystems [6].

Here we discuss global features of multiparty qubit pure states, which can be deduced from their bipartite correlations. We use the property of monogamy of correlations [7]. Another approach has recently been put forward by Würflinger et al. [8] who have shown that some nonentangled reduced density operators can be linked with global entangled states.

Monogamy of quantum correlations can be used for entanglement detection [9]. We use it to derive a criterion for genuine multipartite entanglement. Monogamy has also been employed in studies of the quantum marginal problem, i.e., conditions for the existence of a global quantum state given its marginals [10], in context of the security of quantum key distribution [11], and to show that correlations between macroscopic measurements ought to be classical [12]. It leads to efficient methods of solving strongly correlated multipartite quantum lattice systems [13].

States of $n$ qubits (two-level quantum systems) have density matrices of the following form:

$$
\rho=\frac{1}{2^{n}} \sum_{\mu_{1}, \ldots, \mu_{n}=0,1,2,3} T_{\mu_{1} \ldots \mu_{n}} \sigma_{\mu_{1}} \otimes \cdots \otimes \sigma_{\mu_{n}},
$$

where $\sigma_{\mu_{k}} \in\left\{\mathbb{1}, \sigma_{1}, \sigma_{2}, \sigma_{3}\right\} . \sigma_{1}, \sigma_{2}$, and $\sigma_{3}$ are the Pauli matrices of the $k$ th observer related to the $\hat{x}, \hat{y}$, and $\hat{z}$ local directions of her local Cartesian basis (we allow each observer to choose her own coordinates; this will play a crucial role). The values $T_{\mu_{1} \ldots \mu_{n}}$ are given by a correlation function for measurements of the Pauli operators: $T_{\mu_{1} \ldots \mu_{n}}=\operatorname{Tr}\left(\rho \sigma_{\mu_{1}} \otimes \cdots \otimes \sigma_{\mu_{n}}\right)$. We call the whole object endowed with components $T_{\mu_{1} \ldots \mu_{n}}$ an extended correlation tensor $\hat{T}$. Under rotations of local Cartesian coordinates, its components with $d$ zeros transform like $(n-d)$-order tensors. The values of $T_{i 0 \ldots .}$, i.e., for all indices 0 except $i=1,2$, and 3 for the first qubit indices, give us the components of the Bloch vector $\vec{b}^{1}$ defining the reduced density matrix of the qubit. Similar identifications with Bloch vectors, denoted as $\vec{b}^{(k)}$, hold for all other qubits.

A pure $n$-partite state $|\psi\rangle$ is called a $k$-product state, if it can be represented as a tensor product of $k$ pure $r_{m}$-partite states:

$$
\left|\psi_{k \text {-prod }}\right\rangle=\left|\psi_{r_{1}}\right\rangle \otimes \cdots \otimes\left|\psi_{r_{k}}\right\rangle .
$$

Of course $\sum_{m=1}^{k} r_{m}=n$. There are different types of $k$-product states corresponding to different ways of partitioning $n$ into a sum of $k$ integers. We refer to a definite type of $k$-product state as a $\left(r_{1}+\cdots+r_{k}\right)$-partition product state. For example, an $n=4$ partite state can be 2-product in two ways, $(3+1)$ and $(2+2)$, and it can be 3 -product in one way, $(2+1+1)$. It is clear that if a state is not $k$-product it also cannot be a $k^{\prime}$-product for $k^{\prime} \geqslant k$. If a state is not $k$-product, it can be at most $(k-1)$-product. The number of mutually entangled particles is minimized when entanglement is distributed as uniformly as possible (the maximal number of particles share an entangled state). Thus, a state that is not a $k$-product state has a subset of at least $\lceil n /(k-1)\rceil$ mutually entangled particles. For example, if a 7-partite state is not a 3-product state, it can be at most biseparable. The number of mutually entangled particles is minimal for partition $(3+4)$. Thus, we have entanglement between at least 4 particles.

The quantity which plays the main role in our method is the sum of squares of all possible bipartite correlations. Monogamy relations lead to the following property.

Proposition 1. For any $n$-qubit state (pure or mixed) the following tight bound holds:

$$
\mathcal{M}=\sum_{1 \leqslant k<l \leqslant n} \mathcal{M}_{k l} \leqslant \begin{cases}2 & \text { if } n=2, \\
\left(\begin{array}{l}
n \\
2
\end{array}\right) & \text { if } n \geqslant 3,\end{cases}
$$

with

$$
\mathcal{M}_{k l}=\sum_{i, j=1,2} T_{0, \ldots, 0, i_{(k)}, 0, \ldots, 0, j_{(l)}, 0, \ldots, 0}^{2},
$$

where the subscripts $(k)$ and $(l)$ denote the $k$ th and $l$ th positions, and $i$ and $j$ are two pairs of Cartesian coordinate indices. For simplicity, we assume that they always represent coordinate indices related with directions $x$ and $y$.

Note that $\left(\begin{array}{l}n \\ 2\end{array}\right)$ is the number of terms $\mathcal{M}_{k l}$ in $\mathcal{M}$.

Proof. Our thesis for $n=2$ and $n=3$ follows from the following monogamy relations, which are direct generalizations 
of the ones derived in Ref. [7]:

$$
\begin{gathered}
\mathcal{M}_{k l} \leqslant 2, \quad \text { for all } k \neq l, \\
\mathcal{M}_{k l}+\mathcal{M}_{l m} \leqslant 2, \quad \text { for all } k \neq l \neq m, \\
\mathcal{M}_{k l}+\mathcal{M}_{l m}+\mathcal{M}_{k m} \leqslant 3, \quad \text { for all } k \neq l \neq m .
\end{gathered}
$$

The relations (5) and (6) can be derived using the following property of a set of operators shown in Refs. [7,14,15]: Let $S=\left\{\hat{A}_{1}, \ldots, \hat{A}_{j}\right\}$ be a set of Hermitian, traceless operators fullfilling $\hat{A}_{k} \hat{A}_{l}+\hat{A}_{l} \hat{A}_{k}=0$ and $\hat{A}_{k}^{2}=\mathbb{1}$, and let $\vec{\alpha}=$ $\left(\left\langle\hat{A}_{1}\right\rangle, \ldots,\left\langle\hat{A}_{j}\right\rangle\right)$ be a vector of their expectation values. Then the following holds:

$$
\|\vec{\alpha}\|^{2} \leqslant 1
$$

Without endangering generality we can make a proof of Eq. (5) for just two qubits, say the 1 st and 2nd. Define two sets of operators: $S_{1}=\left\{\sigma_{1} \otimes \sigma_{1}, \sigma_{1} \otimes \sigma_{2}\right\}$ and $S_{2}=\left\{\sigma_{2} \otimes\right.$ $\left.\sigma_{1}, \sigma_{2} \otimes \sigma_{2}\right\}$. It is straightforward to see that the operators within each set fulfill all the above assumptions. However, $\left\|\vec{\alpha}_{1}\right\|^{2}=T_{11}^{2}+T_{12}^{2}$ and $\left\|\vec{\alpha}_{2}\right\|^{2}=T_{21}^{2}+T_{22}^{2}$. Therefore, $\left\|\vec{\alpha}_{1}\right\|^{2}+\left\|\vec{\alpha}_{2}\right\|^{2}=\mathcal{M}_{12} \leqslant 2$.

For the proof of Eq. (6) let us limit ourselves to qubits 1, 2, and 3 , and let us define

$$
S_{1}^{\prime}=\left\{\sigma_{1} \otimes \sigma_{1} \otimes \mathbb{1}, \sigma_{1} \otimes \sigma_{2} \otimes \mathbb{1}, \sigma_{2} \otimes \mathbb{1} \otimes \sigma_{1}, \sigma_{2} \otimes \mathbb{1} \otimes \sigma_{2}\right\},
$$

$S_{2}^{\prime}=\left\{\sigma_{2} \otimes \sigma_{1} \otimes \mathbb{1}, \sigma_{2} \otimes \sigma_{2} \otimes \mathbb{1}, \sigma_{1} \otimes \mathbb{1} \otimes \sigma_{1}, \sigma_{1} \otimes \mathbb{1} \otimes \sigma_{2}\right\}$.

Both sets fulfill assumptions leading to inequality (8); thus after a similar identification of averages of the operators with components of the generalized correlation tensor, we get $\mathcal{M}_{12}+\mathcal{M}_{13} \leqslant 2$.

The inequality (7) follows from summing up the following three inequalities, each of the form of Eq. (6):

$$
\begin{aligned}
& \mathcal{M}_{k l}+\mathcal{M}_{l m} \leqslant 2, \\
& \mathcal{M}_{k l}+\mathcal{M}_{k m} \leqslant 2, \\
& \mathcal{M}_{l m}+\mathcal{M}_{k m} \leqslant 2 .
\end{aligned}
$$

For higher $n$ we combine inequalities (6) and (7) to arrive at the thesis. Let us put $\mathcal{M}$ as follows:

$$
\begin{aligned}
\mathcal{M}= & \left(\mathcal{M}_{12}+\mathcal{M}_{13}+\cdots+\mathcal{M}_{1 n}\right)+\left(\mathcal{M}_{23}+\cdots+\mathcal{M}_{2 n}\right) \\
& +\cdots+\left(\mathcal{M}_{n-2, n-1}+\mathcal{M}_{n-2, n}\right)+\left(\mathcal{M}_{n-1, n}\right),
\end{aligned}
$$

where in every bracket we group the terms that share a common subsystem corresponding to the first index. Consider first the case of even total number of $\mathcal{M}_{k l}$ terms in $\mathcal{M}$, i.e., an even $\left(\begin{array}{c}n \\ 2\end{array}\right)$. In the brackets which contain an even number of $\mathcal{M}_{k l}$ terms, we split the sum into consecutive pairs and apply inequality (6) to every pair. In the brackets with an odd number of $\mathcal{M}_{k l}$ terms, we split the sum into consecutive pairs and the last term. As the total number of $\mathcal{M}_{k l}$ terms is even, the number of "last terms" is also even. The last terms have a common last subsystem. We again group them in pairs. We have $\frac{1}{2}\left(\begin{array}{c}n \\ 2\end{array}\right)$ pairs. By the inequality (6) each pair is upper bounded by 2 . Thus, the sum is bounded by $\left(\begin{array}{l}n \\ 2\end{array}\right)$.
For an odd number of $\mathcal{M}_{k l}$ terms in $\mathcal{M}$, i.e., $\left(\begin{array}{c}n \\ 2\end{array}\right)$ odd, we first apply inequality (7) to its last three terms [in Eq. (12) these terms are shown explicitly]. The number of remaining $\mathcal{M}_{k l}$ terms is even, and we proceed as before: we use $\frac{1}{2}\left[\left(\begin{array}{l}n \\ 2\end{array}\right)-3\right]$ inequalities (6), and one inequality (7). This again gives the bound $\left(\begin{array}{l}n \\ 2\end{array}\right)$.

The bound is tight. Take a state $\otimes_{k=1}^{n}|+\rangle_{k}$, where $|+\rangle_{k}$ is the +1 eigenvalue eigenstate of $\sigma_{x}$ of the $k$ th observer. For the state all $\left(\begin{array}{c}n \\ 2\end{array}\right)$ correlation tensor elements with two 1 indices entering each $\mathcal{M}_{k l}$ are equal to 1 .

Since the bound of $\mathcal{M}$ is attained by a product state, $\mathcal{M}$ seems to be useless as an entanglement identifier. However, this can be overcome by the choice of a suitable local coordinate system for each of the observers. Namely, we say that the $k$ th observer uses her preferred Cartesian basis, if the Bloch vector of her qubit is pointing in the $z$ direction. For such a set of local coordinates the sum in $\mathcal{M}_{k l}$, see Eq. (4), will always be zero for the above-discussed product state, as the local Bloch vectors of the reduced density matrices, which were earlier $\hat{x}$, in the preferred coordinates are by definition $\hat{z}$, and due to the nature of the state, the values entering $\mathcal{M}_{k l}$ factorize. For example, $T_{i j 0 \ldots 0}=T_{i 00 \ldots 0} T_{0 j 0 \ldots 0}=b_{i}^{(1)} b_{j}^{(2)}$. Thus the components for $i=1$ and 2 and $j=1$ and 2 in Eq. (4) are zero.

Let us denote $\mathcal{M}$ in the new preferred set of coordinate systems by $\mathcal{M}^{(\mathrm{pb})}$. It is a sum of bipartite correlations involving only tensor components related to local directions orthogonal to the local Bloch vectors. From now on the local observers do not have any freedom to define the $\hat{z}$ direction-it is set by the Bloch vector of the local reduced density operator. If a given qubit has a vanishing Bloch vector, any axis can serve as the local $\hat{z}$ direction.

The quantity $\mathcal{M}^{(\mathrm{pb})}$ has the following property, which makes it useful as an entanglement identifier.

Proposition 2. For a given type of $k$-product state $\left|\psi_{k \text {-prod }}\right\rangle=$ $\otimes_{m=1, \ldots, k}\left|\psi^{r_{m}}\right\rangle$ the following property holds:

$$
\mathcal{M}^{(\mathrm{pb})}\left(\otimes_{m=1, \ldots, k}\left|\psi^{r_{m}}\right\rangle\right)=\sum_{m=1}^{k} \mathcal{M}^{(\mathrm{pb})}\left(\left|\psi^{r_{m}}\right\rangle\right) .
$$

Proof. Note that in the sum $\mathcal{M}^{(\mathrm{pb})}\left(\left|\psi^{r_{1}}\right\rangle \cdots\left|\psi^{r_{k}}\right\rangle\right)$ correlation tensor elements in formula (4) with index $i$ belonging to one subsystem (say $r_{a}$ ) and index $j$ belonging to another (say $r_{b}$ ) can be factorized, since they are effectively calculated for the product state $\left|\psi^{r_{a}}\right\rangle \otimes\left|\psi^{r_{b}}\right\rangle$. Thus,

$$
T_{0, \ldots, 0, i, 0, \ldots, 0, j, 0, \ldots, 0}=T_{0, \ldots, 0, i, 0, \ldots, 0}^{\left(r_{a}\right)} T_{0, \ldots, 0, j, 0, \ldots, 0}^{\left(r_{b}\right)} .
$$

But the factors on the right-hand side are $x$ or $y$ components of the single-particle Bloch vectors and thus vanish in the preferred Cartesian bases. Thus, correlations between subsystems $r_{a}$ and $r_{b}$ are not present in $\mathcal{M}^{(\mathrm{pb})}\left(\left|\psi^{r_{1}}\right\rangle \cdots\left|\psi^{r_{k}}\right\rangle\right)$. The sum can be decomposed into sums solely within the subsystems from $r_{1}$ to $r_{k}$. That is, we have Eq. (13).

The following proposition is a basis of our method of identifying entanglement.

Proposition 3. For $n \geqslant 3$, for any pure $n$-qubit state $|\psi\rangle$, and for any class $\mathcal{S}$ of $k$-product states of the type $r_{1}+\cdots+r_{k}=n$ 
the following holds:

$$
|\psi\rangle \in \mathcal{S} \Longrightarrow \mathcal{M}^{(\mathrm{pb})}(|\psi\rangle) \leqslant \sum_{m=1}^{k}\left(\begin{array}{c}
r_{m} \\
2
\end{array}\right)+d,
$$

where $d$ is the number of terms in the sequence $r_{1}, \ldots, r_{k}$ with $r_{m}=2$.

Proof. For any state $|\psi\rangle \in \mathcal{S}$ we have

$$
\begin{aligned}
\mathcal{M}^{(\mathrm{pb})}(|\psi\rangle) \leqslant \max _{\left|\psi_{\mathcal{S}}\right\rangle \in \mathcal{S}} \mathcal{M}^{(\mathrm{pb})}\left(\left|\psi_{\mathcal{S}}\right\rangle\right) \\
=\sum_{m=1}^{k} \max _{\left|\psi^{r_{m}}\right\rangle \in \mathcal{H}^{\left(r_{m}\right)}}\left[\mathcal{M}^{(\mathrm{pb})}\left(\left|\psi^{r_{m}}\right\rangle\right)\right] \\
=\sum_{m=1}^{k}\left(\begin{array}{c}
r_{m} \\
2
\end{array}\right)+d,
\end{aligned}
$$

where $\mathcal{H}^{\left(r_{m}\right)}$ is the Hilbert space for subsystems $r_{m}$. The first equality follows from Proposition 2 and the second from Proposition 1. The additional term $d$ comes from the fact that whenever $r_{m}=2$, the maximum of $\mathcal{M}^{(\mathrm{pb})}\left(\left|\psi^{r_{m}}\right\rangle\right)$ is equal to 2 , and $\operatorname{not}\left(\begin{array}{l}2 \\ 2\end{array}\right)=1$.

If $\mathcal{M}^{(\mathrm{pb})}(|\psi\rangle)>\sum_{m=1}^{k}\left(\begin{array}{c}r_{m} \\ 2\end{array}\right)+d$ then we know, thanks to the above criterion, that the state cannot be a $k$-product state with respect to the division $r_{1}, \ldots, r_{k}$. The following lemma can be used to show the maximum of the bound (15) over all possible splittings of $n$ parties into $k$ subsystems, i.e., all $k$-element decompositions of number $n$ into natural numbers.

Lemma 1. For any $n \geqslant 3$, for any $k$ from 2 to $n$, and for any sequence $\left\{r_{m}\right\}_{m=1}^{k}$ such that $\sum_{m=1}^{k} r_{m}=n$, the following conditions hold:

(i) if all $r_{m} \geqslant 1$, one has $\sum_{m=1}^{k}\left(\begin{array}{c}r_{m} \\ 2\end{array}\right) \leqslant\left(\begin{array}{c}n-k+1 \\ 2\end{array}\right)$,

(ii) if all $r_{m} \geqslant 0$, one has $\sum_{m=1}^{k}\left(\begin{array}{c}r_{m} \\ 2\end{array}\right) \leqslant\left(\begin{array}{c}n \\ 2\end{array}\right)$.

Proof. Note that the sum on the left-hand side of the above inequalities can be expressed as

$$
\sum_{m=1}^{k}\left(\begin{array}{c}
r_{m} \\
2
\end{array}\right)=\sum_{m=1}^{k} \frac{r_{m}\left(r_{m}-1\right)}{2}=\frac{1}{2}\left(\sum_{m=1}^{k} r_{m}^{2}-n\right) .
$$

Let us consider the case with all $r_{m} \geqslant 1$. The sum $\sum_{m=1}^{k} r_{m}^{2}$ is maximized when all but one of the terms in the sequence $\left\{r_{m}\right\}_{m=1}^{k}$ are equal to 1 , and the remaining one equals $n-k+$ 1. This can be easily proved using the Lagrange method of finding conditional extrema.

In the case of $0 \leqslant r_{m} \leqslant n$ we can prove that, with given boundary conditions, the sum is maximized when all but one term are 0 , and the one equals $n$.

The results of Lemma 1 and Proposition 3 imply the following corollaries.

Proposition 4. For $n \geqslant 3$, and for any pure $n$-qubit state $|\psi\rangle$, the following implication holds. If $\mathcal{M}^{(\mathrm{pb})}(|\psi\rangle)>s_{k}$ then $|\psi\rangle$ is not $k$-product, where $s_{k}$ for $k=n-1$ is 2 , for $k=$ $n-2$ is 4 , and

$$
s_{k}=\left(\begin{array}{c}
n-k+1 \\
2
\end{array}\right), \quad \text { for } k=2, \ldots, n-3 .
$$

Proposition 5. For $n \geqslant 5$, and for any $n$-qubit pure state $|\psi\rangle$, if

$$
\mathcal{M}^{(\mathrm{pb})}(|\psi\rangle)>\left(\begin{array}{c}
n-1 \\
2
\end{array}\right),
$$

then $|\psi\rangle$ is genuinely $n$-partite entangled. For $n=3$ and $n=4$ we have, respectively,

$\mathcal{M}^{(\mathrm{pb})}(|\psi\rangle)>2 \Longrightarrow|\psi\rangle$ is genuinely 3-partite entangled, $\mathcal{M}^{(\mathrm{pb})}(|\psi\rangle)>4 \Longrightarrow|\psi\rangle$ is genuinely 4-partite entangled.

Our last condition for multipartite entanglement is given by the following proposition.

Proposition 6. For any $n$-qubit pure state $|\psi\rangle$, with $n \geqslant 5$, and for any $m \leqslant\left\lfloor\frac{n}{2}\right\rfloor-1$ the following holds:

$$
\begin{aligned}
& \mathcal{M}^{(\mathrm{pb})}(|\psi\rangle)>\left(\begin{array}{c}
m \\
2
\end{array}\right)+\left(\begin{array}{c}
n-m \\
2
\end{array}\right)+\delta_{m, 2} \\
& \Longrightarrow|\psi\rangle \text { is genuinely } m \text {-partite entangled. }
\end{aligned}
$$

Proof. For any $n$-partite state consider all possible bipartite divisions $\left(r_{1}+r_{2}\right)$, where $r_{1} \leqslant r_{2}$ and $r_{1}+r_{2}=n$. Note that the $\mathcal{M}^{(\mathrm{pb})}$ values corresponding to different divisions are in the following order:

$$
\begin{aligned}
\mathcal{M}^{(\mathrm{pb})}\left(\left|\psi^{1}\right\rangle\left|\psi^{n-1}\right\rangle\right) & >\mathcal{M}^{(\mathrm{pb})}\left(\left|\psi^{2}\right\rangle\left|\psi^{n-2}\right\rangle\right)>\cdots \\
& >\mathcal{M}^{(\mathrm{pb})}\left(\left|\psi^{\lfloor n / 2\rfloor}\right\rangle\left|\psi^{\lceil n / 2\rceil}\right\rangle\right) .
\end{aligned}
$$

Indeed, for any $r_{1} \in\left[1,\left\lfloor\frac{n}{2}\right\rfloor-1\right]$ and $n \geqslant 5$ [such that division $(2+2)$ is excluded] the difference of adjacent divisions is strictly positive:

$$
\begin{aligned}
& \mathcal{M}^{(\mathrm{pb})}\left(\left|\psi^{r_{1}}\right\rangle\left|\psi^{n-r_{1}}\right\rangle\right)-\mathcal{M}^{(\mathrm{pb})}\left(\left|\psi^{r_{1}+1}\right\rangle\left|\psi^{n-r_{1}-1}\right\rangle\right) \\
&=\left(\begin{array}{c}
r_{1} \\
2
\end{array}\right)+\delta_{r_{1}, 2}+\left(\begin{array}{c}
n-r_{1} \\
2
\end{array}\right)+\delta_{n-r_{1}, 2} \\
& \quad-\left(\begin{array}{c}
r_{1}+1 \\
2
\end{array}\right)-\delta_{r_{1}+1,2}-\left(\begin{array}{c}
n-r_{1}-1 \\
2
\end{array}\right)-\delta_{n-r_{1}-1,2} \\
& \geqslant n-2 r_{1}-2>0 .
\end{aligned}
$$

If $\mathcal{M}^{(\mathrm{pb})}(|\psi\rangle)>\mathcal{M}^{(\mathrm{pb})}\left(\left|\psi^{m}\right\rangle\left|\psi^{n-m}\right\rangle\right)$ then, due to the ordering of divisions, the state $\psi$ contains entanglement between at least $m+1$ parties.

As an example of an application of the conditions let us consider a family of $n$-partite Dicke states with $e$ excitations:

$$
\left|D_{n}^{e}\right\rangle=\frac{1}{\sqrt{\left(\begin{array}{l}
n \\
e
\end{array}\right)}} \sum_{\pi}|\pi(\underbrace{1, \ldots, 1}_{e}, \underbrace{0, \ldots, 0}_{n-e})\rangle,
$$

where summation is performed over all combinations. It can be shown that for odd $n$

$$
\mathcal{M}^{(\mathrm{pb})}\left(\left|D_{n}^{e}\right\rangle\right)=\frac{4 e^{2}(n-e)^{2}}{n(n-1)} .
$$

This expression is maximized for states of the type $D_{n}^{(n-1) / 2}$ for which

$$
\mathcal{M}^{(\mathrm{pb})}\left(\left|D_{n}^{(n-1) / 2}\right\rangle\right)=\frac{(n+1)^{2}(n-1)}{4 n} .
$$

Using our conditions for entanglement we can prove the following properties of the family. States $D_{3}^{1}$ and $D_{5}^{2}$ are genuinely multipartite entangled. This follows from Proposition 5. States 
$D_{n}^{(n-1) / 2}$, where $n$ is odd, are genuinely $\left(\frac{n+3}{2}\right)$-partite entangled. This is a consequence of Proposition 6.

The fact that the multipartite entanglement of Dicke states can be detected with lower-order correlations is itself not surprising, since it is known that these states are uniquely determined by their reduced density matrices [16,17]. Namely, a Dicke state with $e$ excitations is the only state compatible with its $2 e$-partite reduced density operators [16,17]. With an increasing $e$ one has a growing dependence on multipartite correlations. It is thus intriguing that the conditions we derive, although solely based on bipartite correlations, detect better entanglement of Dicke states with higher excitation number. One reason for this could be that entanglement is just one of many defining features of a quantum state and for this reason knowledge of the full state is not necessary to detect entanglement.

A different set of conditions based on bipartite correlations, proposed in Ref. [18], uses spin operators. They are linear in correlations whereas ours are quadratic. Quadratic conditions have an advantage: once the bound for entanglement is achieved we can stop measuring as any future measurement can only increase the sum of squared correlations. For the linear conditions all correlations have to be measured because correlations measured in the next experimental setting could be negative.
Note that Dicke states are genuinely $n$-party entangled. The fact that our criterion does not detect this seems to be a drawback. A question emerges: could bipartite correlations be used to detect this entanglement? Note that for a biproduct state there exist two subsystems, say $k$ th and $l$ th with correlations satisfying $T_{i j}=b_{j}^{(k)} b_{j}^{(l)}$ as every correlation is just a product of local averages. Therefore, if a Dicke state were a biproduct state then some of its bipartite correlations would have to satisfy this relation.

We would like to stress that the conditions derived here do not make any additional assumptions about studied states, except purity, and the Dicke states should be treated just as a simple example of their application.

T.P. acknowledges discussions with Minh Cong Tran. The work is supported by the Polish Ministry of Science and Higher Education Grant No. IdP2011 000361. M.M. is supported by the International Ph.D. Project "Physics of Future Quantum-Based Information Technologies" Grant No. MPD/2009-3/4 of the Foundation for Polish Science (FNP). W.L. and M.Ż. are supported by the FNP TEAM project cofinanced by the EU Regional Development Fund. T.P. is supported by the National Research Foundation, the Ministry of Education of Singapore, a NTU start-up grant and NCN Grant No. 2012/05/E/ST2/02352.
[1] R. Horodecki, P. Horodecki, M. Horodecki, and K. Horodecki, Rev. Mod. Phys. 81, 865 (2009).

[2] J.-W. Pan, Z.-B. Chen, C.-Y. Lu, H. Weinfurter, A. Zeilinger, and M. Żukowski, Rev. Mod. Phys. 84, 777 (2012).

[3] D. Kaszlikowski, A. Sen(De), U. Sen, V. Vedral, and A. Winter, Phys. Rev. Lett. 101, 070502 (2008).

[4] C. H. Bennett, A. Grudka, M. Horodecki, P. Horodecki, and R. Horodecki, Phys. Rev. A 83, 012312 (2011).

[5] K. Modi, A. Brodutch, H. Cable, T. Paterek, and V. Vedral, Rev. Mod. Phys. 84, 1655 (2012).

[6] M. Wieśniak, M. Nawareg, and M. Żukowski, Phys. Rev. A 86, 042339 (2012).

[7] P. Kurzyński, T. Paterek, R. Ramanathan, W. Laskowski, and D. Kaszlikowski, Phys. Rev. Lett. 106, 180402 (2011).

[8] L. E. Würflinger, J-D. Bancal, A. Acín, N. Gisin, and T. Vertesi, Phys. Rev. A 86, 032117 (2012).
[9] W. Laskowski, D. Richart, C. Schwemmer, T. Paterek, and H. Weinfurter, Phys. Rev. Lett. 108, 240501 (2012).

[10] A. Klyachko, arXiv:quant-ph/0409113.

[11] V. Scarani and N. Gisin, Phys. Rev. Lett. 87, 117901 (2001); Phys. Rev. A 65, 012311 (2001).

[12] R. Ramanathan, T. Paterek, A. Kay, P. Kurzyński, and D. Kaszlikowski, Phys. Rev. Lett. 107, 060405 (2011).

[13] F. Verstraete, V. Murg, and J. I. Cirac, Adv. Phys. 57, 143 (2008).

[14] G. Tóth and O. Gühne, Phys. Rev. A 72, 022340 (2005).

[15] S. Wehner and A. Winter, J. Math. Phys. (Melville, NY, U.S.) 49, 062105 (2008).

[16] P. Parashar and S. Rana, Phys. Rev. A 80, 012319 (2009).

[17] P. Parashar and S. Rana, J. Phys. A 42, 462003 (2009).

[18] G. Tóth, J. Opt. Soc. Am. B 24, 275 (2007). 